\title{
e-Phaïstos
}

e-Phaïstos

Revue d'histoire des techniques / Journal of the history

of technology

IV-1 | 2015

Les arts de guerre et de grâce (XIVe-XVIIle siècles)

\section{La notion de patrimoine colonial en Chine. Étude de cas théorique et pratique : la ville de Macao}

The notion of colonial cultural heritage in China. A practical and theoretical study: the city of Macao

\section{Monica Jorge Da Costa}

\section{CpenEdition}

Journals

Édition électronique

URL : http://journals.openedition.org/ephaistos/694

DOI : 10.4000/ephaistos.694

ISSN : 2552-0741

Éditeur

IHMC - Institut d'histoire moderne et contemporaine (UMR 8066)

Édition imprimée

Date de publication : 1 avril 2015

Pagination : 118-126

ISSN : 2262-7340

Référence électronique

Monica Jorge Da Costa, «La notion de patrimoine colonial en Chine. Étude de cas théorique et pratique : la ville de Macao », e-Phaïstos [En ligne], IV-1 | 2015, mis en ligne le 24 novembre 2016 consulté le 20 avril 2019. URL : http://journals.openedition.org/ephaistos/694 ; DOI : 10.4000/ ephaistos.694 


\title{
La notion de patrimoine colonial en Chine. Étude de cas théorique et pratique : la ville de Macao
}

\author{
Monica Jorge Da Costa \\ Master en Histoire des techniques, \\ patrimoine et territoires industriels, \\ Université Paris I - Panthéon Sorbonne \\ (France), Université d'Evora (Portugal) \\ et Université de Padoue (Italie).
}

Entreprendre une étude sur l'histoire technique et le patrimoine d'un pays qui nous est méconnu requiert une réflexion approfondie sur les concepts à utiliser et sur la méthode à employer. En effet, un travail scientifique quel qu'il soit s'ancre forcément dans une ligne de pensée, une tradition de recherche du pays d'où le chercheur vient. Toutefois, étudier un autre espace géographique, d'une culture différente, mène le chercheur à se poser de nouvelles questions. Celui-ci est confronté à une nouvelle réalité et doit s'interroger sur sa manière de traiter l'étude. Il peut en effet chercher uniquement à entreprendre une étude à partir des lignes de réflexion et des méthodes européennes, afin d'apporter un nouveau point de comparaison sur des travaux déjà entrepris. Il peut aussi décider de se mettre lui-même en jeu et accepter de remettre en question les méthodes et les réflexions auxquelles il est habitué. Ce second choix, qui fut celui suivi dans mes recherches, mène à une remise en cause de son système de pensée : il faut réapprendre les règles qui s'appliquent au nouvel environnement auquel nous sommes confrontés; il est essentiel de s'adapter pour cerner l'étendue d'un sujet sur une autre culture. Et surtout, le chercheur doit déconstruire un certain nombre de concepts et de fausses certitudes acquises de par son vécu et son environnement culturel habituel, pour aborder dans sa globalité son sujet.

Pourquoi une telle entrée en matière, alors que le sujet traité est celui de la pratique, de l'histoire du bâti et du patrimoine ? Cet article est en réalité une réflexion sur un parcours personnel réalisé. Il présente, sommairement certes, une méthode de recherche basée sur l'ouverture aux méthodes et aux cultures non européennes pour mener une réflexion plus nuancée et plus internationale. De fait, il semble que la méconnaissance de la manière de vivre et de penser d'une autre culture mène souvent les chercheurs européens à des articles scientifiques très bons, mais souvent incomplets et européanocentrés. Prenons un exemple: la notion de patrimoine. En Europe, le patrimoine est le plus souvent associé au "patrimoine bâti », aux monuments historiques classés et préservés. Bien entendu, les recherches récentes tendent à élargir cette définition aux patrimoines immatériels (ex. : le fado, type de musique portugaise classée au patrimoine immatériel de l'UNESCO), aux patrimoines historiques plus récents tels que des usines ou des gares (ex. : le musée d'Orsay, ancienne gare parisienne). Toutefois, la grande majorité des 
européens continuent à penser le patrimoine comme «monuments anciens » tels que les temples d'Athènes, le Colisée de Rome et Notre Dame de Paris. Or, la notion de patrimoine ne possède pas la même signification en Afrique ou en Asie, où la valeur patrimoniale tient plus de la mémoire, de la tradition, des coutumes que de la matérialité de l'objet.

Comment donc un européen peut-il mener une étude patrimoniale sur d'anciens territoires coloniaux, si celui-ci ne prend pas conscience des enjeux existant autour des concepts utilisés ? Plus concrètement, comment réaliser une étude générale et approfondie sur le patrimoine de Macao, ancienne colonie portugaise en Chine, sans s'interroger sur la culture, la conceptualisation du patrimoine et la valeur du passé en Chine ? Enfin, comment mener à bien une analyse de terrain d'un patrimoine colonial comme celui de Macao, fruit de la rencontre de deux cultures dissemblables, ayant une symbolique différente pour des européens et des asiatiques ?

Pour répondre à toutes ces interrogations, notre article s'attardera sur l'exemple de l'étude de la ville de Macao, afin de donner une dimension concrète à notre étude. Pour ce faire, il est nécessaire en amont de faire une analyse conceptuelle de ce qu'est le patrimoine et des différences en matière de son étude en Europe et en Chine, puis nous analyserons les spécificités des enjeux posés par la conservation d'un patrimoine colonial en Chine; enfin, nous aborderons les difficultés d'étude archivistique et de l'analyse de terrain pour ce type de patrimoine.

L'étude d'un patrimoine, quel qu'il soit, appelle dans un premier temps à une réflexion sur le sens à donner à cette notion. Des discussions sur la définition même à donner à « un site culturel patrimonial » ont eu lieu à l'organisation internationale ICOMOS, dont le résultat a été publié le 10 avril 2007. Est défini comme site culturel patrimonial «[toute] localité, paysage, aire d'établissement, complexe architectural, site archéologique, ou structure existante, reconnus ou souvent protégés légalement en tant que site de signification historique et culturelle. ${ }^{1}$. Quant à la notion de «patrimoine culturel» seule, elle se définit comme « l'ensemble des biens, matériels ou immatériels, ayant une importance artistique et/ou historique certaine. ${ }^{2}$.

Poser ces notions permet donc d'aborder une première analyse sur le sens à donner au patrimoine en Europe et en Asie. Il est intéressant de noter que pour ces deux définitions citées ci-dessus, la compréhension en est radicalement différente. En effet, en Europe, nous avons tendance à considérer comme «patrimoine» en priorité le patrimoine matériel : objets plus ou moins anciens (des silex aux machines industrielles), monuments architecturaux, sites archéologiques, aires d'établissements (ex.: usines et mines). Le patrimoine immatériel est lui bien moins représenté, même si des expositions sur des influences musicales, des cultures identitaire et communautaire, voire même sur des gestes ou rites voient le jour. Il semblerait que notre attachement aux objets et aux monuments relève d'une culture de la sauvegarde de l'objet datant pour ce qui concerne la France de la Révolution française et de la disparition violente de monuments et d'objets liés à notre passé et notre histoire. Il est vrai que nous conservons le patrimoine lié à des événements importants de l'histoire ou lié à des personnages importants (ex. : château de Versailles, symbole architectural et résidence principale de Louis XIV). Toutefois, il s'agit là d'une vision patrimoniale très centrée sur la matérialité et l'histoire factuelle, malgré des recherches scientifiques novatrices en matière de patrimoine immatériel et en histoire sociale et culturelle.

Qu'en est-il du patrimoine et de l'histoire en Asie, en particulier en Chine? Pouvons-nous aborder ce même sujet de la même manière? Partons d'une constatation. La Chine est un pays ayant une histoire millénaire, ayant des traces archéologiques et des monuments historiques en nombre considérable. Et pourtant, nos méthodes européennes ne sont pas celles utilisées aujourd'hui en Chine. Essayons de comprendre d'où provient la différence. La première 
est historique, si à la Renaissance en Italie ou à la suite de la Révolution française 3 , les italiens et les français ont pris conscience de l'importance des monuments historiques et des objets d'art qu'ils avaient en leur possession, et de l'intérêt de leur sauvegarde pour le futur, tel n'a pas été le cas de la Chine. À la suite de guerres civiles et de la Seconde Guerre mondiale, la Chine connaît un nouveau régime politique : la République populaire de Chine. Instauré par le parti communiste en 1949, le nouveau régime se crée à partir d'une politique de cohésion sociale centrée sur le parti. Celle-ci s'affirme aux dépens des cultures régionales et de l'histoire des dynasties chinoises précédant le communisme. À cela s'ajoute une importante période de destruction du patrimoine chinois. Outre ces événements historiques, la pensée chinoise est très différente de l'européenne. Il n'est donc pas étonnant qu'une notion telle que le patrimoine soit différente. Si le mot «patrimoine »遗产 (Yíchăn), possède le même sens que le mot en français, littéralement léguer une propriété, la manière de concevoir le legs est lui différent.

Un architecte chinois ayant fait ses études en France a entrepris de réaliser une étude de référence sur la conception du patrimoine en Chine 4 . Celle-ci se base non pas sur la matérialité d'un monument ou d'un objet, mais sur le souvenir de celui-ci, sur sa symbolique. Ainsi, si en Europe nous accordons beaucoup de valeur à la ruine ou au monument original qui doit être conservé à tout prix dans certains cas ; en Chine, cette nécessité n'existe pas, ou en tout cas ne s'exprime pas de la même manière. Comme le dit Liang Zhan dans sa thèse :

«L'écriture ou les objets fragmentaires provoquent le souvenir du destin et des vicissitudes du monument, ils relient les détails pour en constituer le destin historique, pour donner une identité à une nation qui se reflète dans cette histoire. Le monument qui a disparu peut réapparaître dans un autre futur, le temps du souvenir à travers lequel les descendants d'une culture identitaire comme la culture chinoise peuvent sauvegarder leurs propres témoins historiques, qui sont, selon Simon Leys, un fluide perpétuel et ont une valeur figurative. Le passé vit dans le souvenir, et cela est plus important que toute réalité matérielle. »5

L'importance d'un monument n'est pas dans sa matérialité, mais sa fonction et son histoire. Ainsi, un temple chinois peut être détruit et reconstruit plusieurs fois dans son intégralité sur le même site ; sa valeur patrimoniale étant définie par le culte et les rites perpétués dans ce lieu depuis plusieurs siècles, les événements qui s'y sont succédé et le rayonnement de ce temple dans la mémoire populaire. La matérialité du temple n'est, elle, que secondaire; le monument originel peut être conservée et valoriser si le monument à cette importance mémorielle, mais ce n'est pas systématique.

Au-delà de cet aspect mémoriel essentiel dans la pensée patrimoniale chinoise, il est utile de faire un point sur l'importance du lien nature-culture au niveau du patrimoine en Chine. De fait, une grande partie des sites classés au patrimoine mondial en Chine se trouve dans des sites naturels d'exception. La pensée chinoise reposant en grande partie sur le Taoïsme et le Bouddhisme, l'homme est souvent considéré comme faisant partie d'un tout (le Dao pour le Laozi) ${ }^{6}$, dans lequel il s'inscrit en harmonie avec ce qui l'entoure. Ainsi, les principaux temples chinois sont pour une grande majorité situés au niveau des monts, en lien direct avec la nature. Il est donc plus aisé pour un chinois de considérer comme patrimoine un espace mélangeant monument, élément naturel et mémoire que pour un européen habitué à associer un monument historique à des événements factuels, son architecte et un style esthétique.

Ces différences de conception nous obligent donc à faire des choix pour pouvoir étudier le patrimoine colonial. Devons-nous le traiter selon les définitions européennes ou selon le concept chinois ? Le choix posé ici est de s'intéresser au patrimoine colonial portugais en Chine à Macao, à travers l'étude de l'avenue principale de la ville (l'avenue Almeida Ribeiro). Le choix posé a été, dans la mesure du possible, de mener une étude européenne (donc matérielle, centrée sur le patrimoine bâti et sa 
construction) en y additionnant une réflexion sur la culture chinoise et surtout sur l'usage des lieux afin de comprendre réellement le sens de ce patrimoine pour les chinois d'aujourd'hui (symbole, passé représenté, valeur donnée aux monuments...).

La réalisation de cette étude patrimoniale permet de monter des enjeux actuels politiques et de récupération mémorielle. De fait, Macao, ville en Chine classée au patrimoine mondial de l'UNESCO, est une ville portuaire construite par les portugais dès 1523. Sa conservation symbolise la rencontre de l'occident et de l'orient en Asie. Cette conservation est donc justifiée de par le patrimoine colonial unique de Macao en Chine. Pourtant, ce patrimoine est aussi représentatif du " siècle de la honte ${ }^{7}$ pour la majorité des chinois.

En effet, pour un occidental, Macao symbolise la grandeur des voyages maritimes ayant permis aux navigateurs aventuriers d'atteindre des pays situés à l'autre extrémité du globe. De plus, la trace de cette présence symbolise une histoire longue de cinq siècles de présence portugaise en Chine, globalement pacifique, malgré quelques incidents. La présence de « comptoirs » n'a pas la même signification qu'une présence coloniale impérialiste. Il est important de distinguer la présence portugaise en Chine de celle au Brésil ou en Afrique, beaucoup plus territoriale et impérialiste. De fait, dès les premières installations officielles, les dirigeants chinois sont vus comme étant des acteurs politiques avec lesquels les portugais se doivent de collaborer pour assurer leur présence. La vision du patrimoine colonial de Macao est donc vue par les européens, en particulier portugais, comme une présence plutôt positive et essentiellement commerciale en Chine. L'étude patrimoniale, essentiellement centrée sur l'architecture et les monuments historiques, est une manière de valoriser un passé glorieux sans réellement provoquer un conflit moral pour les historiens et les conservateurs européens. La conservation du patrimoine pour les européens de Macao marque surtout une volonté de souvenir et de sauvegarde matérielle de ce que la nation portugaise a réalisé par le passé, s'inscrivant dans la même lignée que la volonté de conservation d'un régime administratif spécial de Hong-Kong par les anglais. La conservation est donc un moyen de préserver une certaine trace de la présence européenne en Chine pour les occidentaux.

Néanmoins, cette vision de Macao n'est clairement pas celle de la Chine ni celle des habitants de Macao, et pour cause. Pour la Chine, la présence d'européens sur leur territoire a été et est toujours considérée comme une "honte" pour le pays. Il s'agit là d'une présence étrangère non désirée, mais imposée par des traités dits «inégaux ». Il s'agit donc d'une véritable colonisation pour eux. La conservation de ce patrimoine est donc le symbole dans une certaine mesure de cette présence européenne. Ce qui est intéressant de noter est que le patrimoine de Macao ne s'inscrit pas uniquement dans cette vision "anticolonialiste ", mais aussi dans un souci de récupération d'image d'un patrimoine unique et d'une nouvelle publicité politique pour les chinois. En premier lieu, la Chine mène une politique de communication, utilisant son histoire et «son patrimoine» comme l'un des moyens de publicité tant pour l'occident que pour sa propre population ${ }^{8}$. De quoi s'agit-il au juste? L'exemple de Shanghai et de son quartier dit «traditionnel» montre clairement cette politique. Ce quartier, construit dans les années 1950, est aujourd'hui l'un des lieux les plus visités de la ville. Il s'agit d'une construction-imitation récente d'un village ancien typique des environs de Shanghai, le but étant de donner un caractère, un point de repère chinois à la ville en réponse à la présence européenne. Il s'agit surtout d'un lieu de reconstruction historique puisque aucun village de ce type n'a existé dans cette ville. La Chine chercherait à créer une vitrine de ce qu'est être chinois, dans une ville marquée par une présence occidentale. Il en va de même pour Macao. La construction de nombreux casinos modernes donne une nouvelle image à la ville, image de réussite « à la chinoise». Quant au patrimoine portugais et 
notamment l'avenue Almeida Ribeiro, il s'agit surtout de maintenir une image de Chine occidentalisée ouverte vers l'Europe à des fins politiques. Le cœur historique portugais, considéré

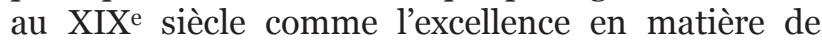
modernité, fait face aujourd'hui à la nouvelle ville, constituée de casinos et d'immeubles rivalisant de hauteur, symbole de cette nouvelle Chine développée et «moderne». Quant à la signification du patrimoine historique de Macao pour les chinois d'aujourd'hui, il s'agit surtout de la visite touristique d'une ville différente, le plus souvent entre deux parties de jeux; car si la plupart visitent le cœur historique, les véritables attractions sont les casinos, leurs salles de jeu, les discothèques et les centres commerciaux de luxe.

Attardons-nous maintenant sur la signification du patrimoine colonial de Macao pour la population locale. De fait, la perception du patrimoine de Macao pour un chinois « mainland »9 ou un macanéen n'est pas tout à fait la même ; le premier regardant Macao comme une ville étrange, pas tout à fait chinoise ; l'autre en y apposant ses repères, son vécu et son environnement. De fait, la perception du patrimoine et de sa valeur identitaire posent question en ce qui concerne la population actuelle de la ville. La sauvegarde du patrimoine est un moyen pour la population de préserver une trace d'un passé, dans un pays en constant développement urbain, les villes changeant de visage tous les dix ans ${ }^{10}$ (destruction et reconstruction constante). Il s'agit aussi d'affirmer une identité différente de celle des "mainlands », nombreux aujourd'hui à Macao, qui ne possèdent pas les mêmes codes culturels et les mêmes manières de vivre que les macanéens. ${ }^{11}$ De plus, le maintien d'une identité locale différente, sans tomber dans une nostalgie coloniale qui est combattue à Macao, permet à la ville de continuer à justifier politiquement son statut particulier de R.A.S. (Région à Administration Spéciale). Ainsi, le patrimoine à Macao est vu par la population comme un moyen d'affirmer une différence vis-à-vis de la Chine, une histoire unique à travers un patrimoine hybride qui n'est ni réellement portugais ni réellement chinois, ainsi qu'un domaine rentable en matière d'économie locale puisque la ville vit essentiellement de l'activité touristique, qu'il s'agisse de tourisme de jeu ou de tourisme patrimonial.

À la suite de ces considérations d'ordre conceptuel, passons à la méthode employée pour le cas de Macao. Il s'agit ici d'analyser la méthode de recherche utilisée dans le cadre d'un master sur le patrimoine colonial de Macao, présenté en soutenance en septembre 2014. Il s'agit d'une étude de cas spécifique du patrimoine de Macao : l'avenue Almeida Ribeiro. Ce type d'aménagement hybride nécessite une approche novatrice, afin de s'adapter tant aux conceptions européennes et chinoises du patrimoine, mais aussi de manière à traiter tant dans la matérialité que dans l'immatérialité un patrimoine colonial sujet à controverse.

Avant d'entamer toute étude patrimoine de terrain, il est nécessaire de se pencher sur le passé de cette avenue, constituée de différents immeubles (dont certains classés comme monuments historiques de la ville), encadrant un axe principal de circulation. L'étude débute donc sur l'origine et la construction de ce patrimoine, et donc commence à partir d'une recherche aux archives. La particularité d'une étude sur une ville coloniale est que ces archives ne sont ni conservées aux mêmes centres archivistiques, ni dans le même pays, voire dans ce cas, ni même sur le même continent. En ce qui concerne les archives en Europe, il est relativement aisé d'y accéder. Toutefois, accéder aux archives à Macao a été dans une certaine mesure une épreuve. En effet, si le centre archivistique de Macao possède toutes les qualités pour conserver correctement les archives, le personnel en charge de mettre à disposition le matériel archivistique aux chercheurs n'est pas correctement formé, la majorité ne parlant pas le portugais, langue pourtant la plus utilisée dans les archives. Les requêtes s'avèrent difficiles tant par leur méconnaissance des archives non chinoises (qui constituent la grande majorité des archives conservées) que par leur niveau d'anglais 
assez variables. Néanmoins, après plusieurs malentendus, il a été possible d'accéder aux documents demandés. Il serait cependant dommage que, pour des questions de langage, ce type d'archives soit méconnu.

Les recherches en archives ont révélé le caractère très technique du patrimoine étudié, notamment par la présence de plans d'architectures, de dossiers de demande de construction, de visites de chantier ou de mémoires sur les matériaux utilisés. De fait, l'étude patrimoniale s'est organisée autour de plusieurs axes : l'étude historique de la construction du bâti, l'étude de l'évolution de l'avenue sur une période donnée, mais aussi de son usage. Pour ce faire, l'étude de cas a été réalisée grâce à une base de données de photographies prises sur le terrain, sur l'analyse des constructions originales grâce aux plans, ainsi que l'étude des matériaux utilisés et des innovations techniques de la période. L'étude de la conservation actuelle du patrimoine intervient après une analyse spatiale et anthropologique de l'avenue étudiée lors de sa construction : type de bâtiments, usages, populations... L'ensemble de cette étude historique permet de comprendre les enjeux actuels de la conservation, à savoir les espaces protégés et ceux soumis aux politiques de réaménagement constant de la ville, les problèmes liés aux commerces touristiques et la modification des bâtiments pour les besoins de publicité et de fonctionnalité...

Ainsi, ce travail est pensé sur plusieurs niveaux de réflexion. Dans un premier temps, il s'agit de faire une étude concrète du patrimoine, qui se différencie des travaux réalisés précédemment à Macao, essentiellement d'histoire plus traditionnelle ou d'architecture (pour ce qui concerne le patrimoine). En outre, ce travail se veut tant pratique que théorique. La pratique permet de comprendre l'histoire technique au moyen de l'étude des matériaux et des constructions luso-chinoises, mais aussi les problèmes liés à la conservation concrète des monuments aujourd'hui. L'étude plus théorique et conceptuelle a pour but de comprendre la culture de Macao ni tout à fait chinoise ni tout à fait portugaise, afin d'écrire une étude permettant un dialogue commun entre chinois, macanéens et portugais sur le patrimoine colonial de Macao, de manière à réaliser une conservation et une valorisation qui respecte et implique chacun de ces différents acteurs. Ce type d'étude permet une approche à la fois éthique et émique ${ }^{12}$ permet d'intégrer dans des études européennes, basée sur l'histoire technique, l'architecture et l'histoire, une analyse de la perception de l'espace par les chinois, une réflexion sur leur mode de vie et sur leur espace de sociabilité.

Cette recherche s'inscrit donc dans une réflexion sur une étude internationale du patrimoine et sur les problèmes rencontrés tant en matière de définition de ce qui « est patrimoine » que de la manière de le considérer selon les cultures. L'étude de cas de Macao constitue un exemple parmi d'autres de ce qui peut être réalisé dans les limites de notre réflexion européenne. Il s'agit donc ici d'exposer des méthodes d'analyse de terrain, des axes de recherche ouverts sur d'autres disciplines dans un souci d'étude générale (anthropologie, urbanisme, histoire...). Cette étude permet aussi d'avoir une vision plus large de la définition du mot « patrimoine », de manière à donner de nouvelles pistes de réflexion, pour envisager à plus long terme des études patrimoniales multidisciplinaires et internationales.

1 Comité scientifique ICOMOS "Charte ICOMOS Pour l'interprétation et la présentation des sites culturels patrimoniaux », 10 avril 2007,

http://www.icomos.org/icahm/documents/Charte_ICOMOS_Int erpretation_FR_10-04-07.pdf

2 Définition tirée du Dictionnaire Robert.

3 GUILHAUMOU, Jacques, "La Révolution française et l'intelligence politique de l'héritage historique: les objets patrimoniaux ", Recensions, Révolution Française.net, mis en ligne le 25 octobre 2007, http://revolution- 
francaise.net/2007/10/25/112-lrevolution-intelligence-politiqueheritage-objets-patrimoniaux.

4 ZHAN, Liang, La naissance du concept de patrimoine en Chine $X I X^{e}-X X^{e}$ siècles, Paris, IPRAUS, 2003.

5 ZHAN, Liang, La naissance du concept de patrimoine en Chine $\mathrm{XIX}^{\mathrm{e}}-\mathrm{XX}^{\mathrm{e}}$ siècles, Paris, IPRAUS, 2003, p.23.

6 DESPEUX, Catherine, Lao-Tseu, le guide de linsondable, édition entrelacs, 2010, p.61 à 72.

7 Expression de SANJUAN, Thierry, TROLLIET, Pierre, La Chine et le monde chinois: une géopolitique des territoires, Paris, Armand Colin, 2010. p.59.

8 Cette idée est particulièrement défendue par SANJUAN, Thierry et TROLLIET, Pierre, dans leur ouvrage La Chine et le monde chinois : une géopolitique des territoires, Paris, Armand Colin, 2010.p.146.

9 Ce terme « mainland » est une expression très utilisée à Macao pour désigner les chinois provenant de Chine continentale, en comparaison avec les habitants des R.S.A (Hong-Kong, Macao).

10 À titre d'exemple, la ville de Macao et l'île de Taipa voisine ont connu en l'espace de dix ans une importante modification urbaine avec la création de nombreux casinos tels que le Venitian, le Sand, le Galaxy ou encore le Grand Lisboa. Des constructions de la même ampleur sont en cours actuellement sur les îles de Taipa et de Coloane modifiant radicalement le paysage.

11 HOPE CLAYTON, Cathryn, "Discourse on the city : identity formation and urban change in contemporary Macao ", Revista de Cultura, $\mathrm{n}^{\circ} 3$, 2002, p.59-81

12 DE SARDAN, Jean-Pierre Olivier, «Émique », L'Homme, 1998 , tome $38 \mathrm{n}^{\circ} 147$, p. 151-166. 


\section{Bibliographie}

\section{- Ouvrages généraux}

ALVES, Paula, BELTRÃO COELHO, Rogério, JORGE, Cecília, Cem anos que mudaram Macau, Macao, Edição do Governo de Macau, 1995.

AMARO, Ana Maria, Das Cabanas de palhas as torres de betão, Lisbonne, Universidade técnica de Lisboa, 1998.

CAÇORINO DA PALMA BARACHO, Carlos Alberto, Um percurso ao encontro do medieval em Macau: urbanismo e arquitectura: persistência $e$ coexistência de elementos medievais portugueses $e$ tradicionais chineses na estrutura urbana $e$ arquitectónica de Macau nos séculos XVI e XVII, Lisbonne, Université Nouvelle de Lisbonne, 1992.

DIAS, Pedro, A Urbanisação e a arquitectura dos portugueses em Macau: 1557 - 1911, Portugal Telecom Lisbonne, Lisbonne, 2005.

GRENET, Jacques, Le Monde Chinois : l'époque contemporaine, Vol.3, Paris, Armand Colin, 2006.

MATTOSO, José (dir.), ROSSA, Walter (Cord.), Património de origem portuguesa no Mundo: arquitectura e urbanismo, Vol. 3 Ásia Oceania, Lisbonne Fondação Calouste Gulbenkian, 2010.

\section{- Ouvrages spécialisés}

CALADO, Maria, Dossier de Candidatura à Lista do Património Mundial UNESCO, Macao, Centro Histórico de Macau, 1987.

DE ARAUJO GOMES DA COSTA, Georgia Manuela, Património Cultural de Macau: a réutilização como instrumento de reabilitação, Lisbonne, Université Technique de Lisbonne, 2005.
DE SARDAN, Jean-Pierre Olivier, «Émique », L'Homme, 1998, tome $38 \mathrm{n}^{\circ} 147$, p.151-166.

INSTITUTO CULTURAL DE MACAU, International Symposium on the Culture of Metropolis in Macau: Modernity, Modernism and Modernization. Urbanism, Identity, Collective Heritage and Quotidian culture - Strategies for the $21^{\text {st }}$ Century, September 1998, Macao, Instituto Cultural de Macau. Macao, Instituto Cultural de Macau, 2001.

JORGE DA COSTA, Monica, L'Urbanisation à Macao: entre traditions et modernité. D'un renouveau urbain à la ville d'aujourd'hui (mémoire de master), soutenue à Paris, Université Paris I Panthéon Sorbonne, 2014.

SANJUAN, Thierry, TROLLIET, Pierre, La Chine et le monde chinois : une géopolitique des territoires, Paris, Armand Colin, 2010.

SANJUAN, Thierry, À l'ombre de Hong-kong, le delta de la Rivière des Perles, Paris, L'Harmattan, 1997.

ZHAN, Liang, La naissance du concept de patrimoine en Chine XIXe - XXe siècles, Paris, IPRAUS, 2003.

\section{- Articles spécialisés}

BURNAY, Diogo, «Mutações e arquitecturas: arquitectura e colonialismo em Macau », Revista de Cultura de Macau, $\mathrm{n}^{\circ} 35 / 36$, Série II, 1998, p.95106.

CALADO, Maria, MENDES, Maria Clara, TOUSSANT, Michel, « Da Fundaçõ aos anos 70: Evolução sócio-económica, urbana e arquitectónica », Revista de Cultura de Macau, $\mathrm{n}^{\circ} 34$, Série II, 1998, p.75-146. 
CODY, Jeffrey, "Cutting Fabric - Macau's San Ma Lo (Avenida Almeida Ribeiro, 1910- 1922)», Dialogue, Architecture + Design + Culture, $\mathrm{n}^{\circ} 30$, 1999, p.52-59.

GUNN, Geoffrey, MICHELON, Laurent, « De quelques ambitions internationales », Perspectives Chinoises, $\mathrm{n}^{\circ}$ 55, 1999, p.43-50.

GOVERNO DE MACAU, «Legislação sobre património », Revista da Cultura de Macau, $\mathrm{n}^{\circ}$ 35/36, Série II, 1998, p233-262.

GUILHAUMOU, Jacques, « La Révolution française et l'intelligence politique de l'héritage historique : les objets patrimoniaux », Recensions, Révolution Française.net, mis en ligne le 25 octobre 2007 , URL:http://revolutionfrancaise.net/2007/10/25/112-lrevolutionintelligence-politique-heritage-objetspatrimoniaux.

RODRIGUES COSTA, Maria de Lourdes, " História da arquitectura em Macau », Revista de Cultura de Macau, n ${ }^{\circ}$ 34, Série II, 1998, p. 181-240.

HOPE CLAYTON, Cathryn, "Discourse on the city: identity formation and urban change in contemporary Macao», Revista de Cultura de Macau, edição internacional, ${ }^{\circ} 3$, 2002, p.59-81.

LECLERC, Alain, «Recomposition de la tradition dans les espaces familiaux et urbains en Chine ", (à paraître), 11 pages.

SALA, Ilaria Maria, « De nouveaux musées pour une nouvelle histoire », Perspectives Chinoises, $\mathrm{n}^{\circ} 51,1999, \mathrm{p} .62-70$.

VIDEIRA PIRES, Benjamin, "A "Cidade China" há cem anos ", Revista de Cultura de Macau, $\mathrm{n}^{\circ} 7$ et $8,1988-1989$, p.55-62. 